\title{
Implications of microRNA-197 \\ downregulated expression in esophageal cancer with poor prognosis
}

\author{
T.-Y. Wang1, S.-G. Liu' ${ }^{2}$, B.-S. Zhao ${ }^{2}$, B. Qi², X.-G. Qin² and W.-J. Yao \\ ${ }^{1}$ Department of Biochemistry and Molecular Biology, \\ Xinxiang Medical University, Henan, China \\ ${ }^{2}$ Department of Thoracic Surgery, \\ The First Affiliated Hospital of Xinxiang Medical University, Weihui, China \\ Corresponding author: T.-Y. Wang \\ E-mail: wtianyuncn@126.com
}

Genet. Mol. Res. 13 (3): 5574-5581 (2014)

Received July 11, 2013

Accepted December 9, 2013

Published July 25, 2014

DOI http://dx.doi.org/10.4238/2014.July.25.12

\begin{abstract}
The aim of this study was to investigate the significance of the microRNA miR-197 expression level in relation to clinicopathological factors and prognoses of esophageal cancer (EC). MicroRNA was extracted using the Taqman ${ }^{\circledR}$ MicroRNA Assay from 46 EC patients at the same tumor node metastasis (TNM) stage, but with different prognoses, who underwent surgery. Paracancerous normal tissues were used as controls. The correlation between miR197 expression and clinicopathologic features was analyzed, and the significance of miR-197 as a prognostic factor and its relationship with survival was determined. miR-197 expression was lower in patients with poor prognosis than in those with good prognosis $(\mathrm{P}<0.05)$. KaplanMeier analysis results showed that the miR-197 expression level is significantly correlated with survival time $(\mathrm{P}=0.030)$, and that patients with higher expression of miR-197 had longer survival times. Cox multi-factor model analysis showed that patient prognosis $(\mathrm{P}=0.001)$, tumor length $(\mathrm{P}=0.010)$ and expression $(\mathrm{P}=0.042)$, and survival time
\end{abstract}


were significantly correlated, with corresponding risks of 9.183, 2.318, and 1.925 , respectively. This study supports a role of miR-197 as an anti-oncogene and a biomarker for EC and its relationship with other prognostic factors and survival.

Key words: Esophageal cancer; MicroRNA-197; Prognosis

\section{INTRODUCTION}

Esophageal cancer (EC) is one of the most prevalent cancers, and its incidence continues to increase, resulting in major concerns regarding human health. Various modalities have been attempted for EC treatment, and the mortality rate is decreasing with medical advances. However, outcomes in EC patients are variable even in patients in the same stage. To individualize treatment and to predict outcomes, novel biomarkers for the development of molecular diagnostic tests are required.

MicroRNAs (miRNAs), first discovered in 1993 (Lee et al., 1993), are small noncoding, endogenous RNA gene products consisting of 18-25 nucleotides encoded by specific genes (Ambros, 2004; Kim, 2005; Hobert, 2008). After a multistage process commencing in the nucleus, pri-mRNAs are trimmed into pre-mRNAs and transported into the cytoplasm. Following cleavage by the endoribonuclease dicer, pre-miRNAs are converted into mature miRNAs that are incorporated into RNA-induced silencing complex (miRISC) and target messenger RNA (mRNA), resulting in cleavage or translational repression. Typically, miRNAs bind to the 3'-untranslated region (UTR) of target mRNAs and decrease the abundance of target proteins. The effect is mediated mainly by translational repression (Meister, 2007; Filipowicz et al., 2008) and, in some cases, decreases in mRNA abundance (Bagga et al., 2005; Lim et al., 2005). Numerous studies have established miRNAs as broad and powerful regulators of protein expression in physiology and disease (Liang et al., 2009; Liang, 2009).

Recent studies have found that miRNAs affect both oncogenes and anti-oncogenes, and play important roles in the initiation and development of tumors (Liang et al., 2009; Liang, 2009). In addition, miRNA spectra are correlated with the diagnosis, staging, progression, and prognosis of EC (Zhou et al., 2009; He et al., 2012; Yang et al., 2013b). Studies using microarray technology to examine the miRNA expression spectra of $31 \mathrm{EC}$ tissue samples revealed that 46 miRNAs exhibit abnormal expression, 7 of which exhibit significant differences compared with those in normal tissues; furthermore, high expression of has-miR-103/107 is closely correlated with low survival rate. To date, has-miR-335, has-miR-181d, has-miR-25, has-miR-7, and has-miR-495 have been confirmed to be correlated with histopathological stages of EC. In addition, abnormal miRNA expression spectra differ between esophageal squamous cell carcinoma (ESCC) tissues and esophageal adenocarcinoma (EAC) tissues (Guo et al., 2008; Mathé et al., 2009; Lin et al., 2012; Xu et al., 2012).

In clinical practice, we found that EC patients at same pathological stage that received the same surgical therapy by the same surgeon had distinctive prognoses. Whether differences in miRNA expression among these patients contributed to the different postoperative prognoses remains unclear.

In our previous study, we found that miR-197 expression was related to the prognosis of ES patients who received the same therapy at the same pathological stage. The present study was performed to determine the biological role of miR-197 expression in EC by inves- 
tigating the relationship between miR-197 expression and clinicopathological characteristics and survival in EC patients.

\section{MATERIAL AND METHODS}

\section{Tissue samples}

We collected 46 clinical and pathologic reports of patients at the same pathological stage but with different prognoses at stages I-III who received treatment at the First Affiliated Hospital of Xinxiang Medical University between September 2006 and December 2009. Among the 46 patients, 18 had good prognoses while the other 28 cases had poor prognoses. In addition, paracancerous normal esophageal mucous membranes $8 \mathrm{~cm}$ distant to the verge of the tumor tissue were used as controls. This study was conducted in accordance with the Declaration of Helsinki and with approval from the Ethics Committee of Xinxiang Medical University. Written informed consent was obtained from all participants.

\section{Sample treatment and RNA extraction}

Using a microtome, $10-\mu \mathrm{m}$ slices of formalin-fixed, paraffin-embedded tissues were obtained and placed in $1.5-\mathrm{mL}$ microcentrifuge tubes. Total RNA was extracted using TRI Reagent (Applied Biosystems; Foster City, CA, USA) according to manufacturer instructions. Briefly, $0.75 \mathrm{~mL}$ TRI Reagent was added and then incubated at room temperature for $5 \mathrm{~min}$. After adding $0.5 \mathrm{~mL}$ isopropanol and mixing well, samples were stored for $2 \mathrm{~h}$ at $-20^{\circ} \mathrm{C}$ and centrifuged at $13,000 \mathrm{~g}$ for $15 \mathrm{~min}$ at $4^{\circ} \mathrm{C}$. The supernatant was removed and $1 \mathrm{~mL} 70 \%$ icecold ethanol was added to dissolve the pellet. The sample was re-centrifuged and the supernatant was discarded. The final pellet was dissolved in $50 \mu \mathrm{L}$ diethylpyrocarbonate distilled water, and the RNA yield was determined using an ultraviolet (UV) spectrophotometer.

\section{Real-time quantitative reverse transcription-polymerase chain reaction (RT-qPCR)}

A total of $0.5-1 \mu \mathrm{g}$ RNA was used in the reverse transcriptase reaction. After reverse transcription, a TaqMan MicroRNA Assay (Applied Biosystems) was performed according to the manufacturer protocol to detect miR-197expression using miR-197 as a primer; the small nRNA RNU6 (Assay ID: 001093) was used as a control. The relative quantitative method was used. Gene expression was quantitated based on the following formula: $\mathrm{F}=2^{-\Delta \Delta \mathrm{Ct}}$ where $\Delta \Delta \mathrm{Ct}=$ [mean cycle threshold $(\mathrm{Ct})$ of the target gene in the test sample - mean $\mathrm{Ct}$ of the housekeeping gene in the test sample] - (mean Ct of the target gene in the control sample - mean Ct of the housekeeping gene in the control sample). A higher F-value indicates relatively higher expression.

\section{Statistical analysis}

The relationship between the miR-197 expression level and clinicopathologic features of the patients was analyzed using the chi square test. We used SPSS17.0 (SPSS, Inc.; Chicago, IL, USA) to establish and analyze the data. The Kaplan-Meier method was used to analyze the relationship between miRNA expression and survival time. A Cox regression model 
was used to analyze the influence of the related factors on survival time of patients with EC. $\mathrm{P}<0.05$ was considered to be statistically significant.

\section{RESULTS}

\section{Patient characteristics}

Forty-six patients with ESCC (without preoperative radiotherapy and chemotherapy) were enrolled in this study. All patients underwent surgical resection at the Department of Thoracic Surgery of the First Affiliated Hospital of Xinxiang Medical University. All postoperative samples were confirmed for ESCC by histopathological examination. Tumors were staged according to the International Union against Cancer (UICC) staging criteria for esophageal carcinoma, (Sobin et al., 2002). The study patients consisted of 23 males and 23 females aged 45-71 years (median, 59 years). Nineteen tumors were $>5 \mathrm{~cm}$ and 27 were $\leq 5 \mathrm{~cm} ; 11$ cases showed good tumor differentiation, 19 cases showed intermediate differentiation, and 16 cases showed poor differentiation. Nineteen cases showed tumor lymph node metastasis, and 27 cases were not metastatic. One case was at stage 0,6 cases were in stage I, 20 cases were in stage IIa, 1 cases was in stage IIb, and 18 cases were in stage III. There were 28 early recurrence patients (tumor recurrence within 1 year after operation) and 18 non-recurrence patients (patients who survived more than 5 years without recurrence). The patient characteristics are outlined in Table 1.

\begin{tabular}{|c|c|}
\hline Characteristics & No. of patients (\%) \\
\hline All Patients & $46(100.0)$ \\
\hline \multicolumn{2}{|l|}{ Gender } \\
\hline Male & $23(50.0)$ \\
\hline Female & $23(50.0)$ \\
\hline \multicolumn{2}{|l|}{ Age } \\
\hline$\geq 60$ years & $21(45.7)$ \\
\hline$<60$ years & $25(54.3)$ \\
\hline \multicolumn{2}{|l|}{ Site of tumor } \\
\hline Upper thoracic & $4(8.7)$ \\
\hline Middle thoracic & $35(76.1)$ \\
\hline Lower thoracic & $7(15.2)$ \\
\hline \multicolumn{2}{|l|}{ Size of tumor } \\
\hline$\leq 5 \mathrm{~cm}$ & $27(58.7)$ \\
\hline$>5 \mathrm{~cm}$ & $19(41.3)$ \\
\hline \multicolumn{2}{|l|}{ Differentiation } \\
\hline Well & $11(16.3)$ \\
\hline Moderate & $19(41.3)$ \\
\hline Poor & $16(34.8)$ \\
\hline \multicolumn{2}{|c|}{ Lymph node metastasis } \\
\hline Negative & $27(58.7)$ \\
\hline Positive & $19(41.3)$ \\
\hline \multicolumn{2}{|l|}{ Depth of invasion } \\
\hline Tis, $\mathrm{T} 1$ & $8(17.4)$ \\
\hline $\mathrm{T} 2$ & $6(13.0)$ \\
\hline T3 & $32(69.6)$ \\
\hline \multicolumn{2}{|l|}{ Stage (TNM) } \\
\hline $0-\mathrm{I}$ & $7(15.2)$ \\
\hline IIa-IIb & $21(45.7)$ \\
\hline III & $18(39.1)$ \\
\hline \multicolumn{2}{|l|}{ Recurence } \\
\hline Early recurence & $28(60.9)$ \\
\hline Non-recurence & $18(39.1)$ \\
\hline
\end{tabular}




\section{miR-197 expression}

The has-miR-197 expression level increased 5.905-fold in samples with good prognoses compared with samples with poor prognoses. The mean expression level of the samples with good prognoses was $4.768 \pm 3.046$, while the mean expression level was $2.501 \pm 3.327$ in the samples with poor prognoses; the difference between these samples was statistically significant (Figure $1 \mathrm{~A}$ and $\mathrm{B} ; \mathrm{P}<0.05$ ).

A

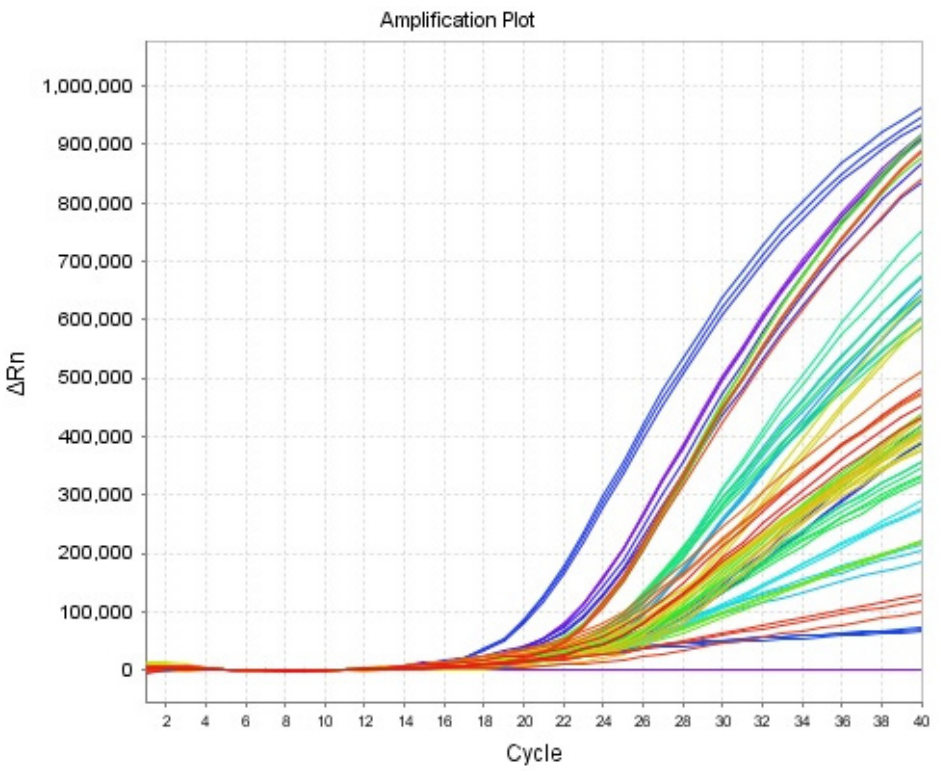

B

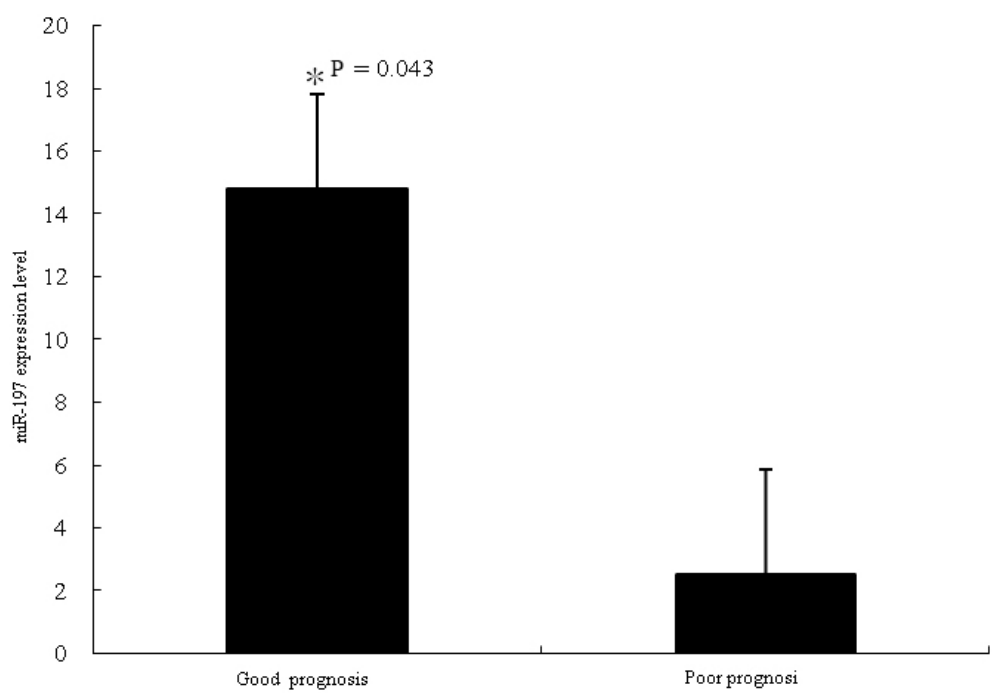

Figure 1. A. Amplification plot with $\geq \mathrm{Ct}$ values of miR-197 in esophageal cancer with good and poor prognosis; B. Comparison of miR-197 expression in esophageal cancer between the sample with good and poor prognosis. 


\section{Relationship between miR-197 expression and prognosis and survival of EC}

The Kaplan-Meier analysis results showed that the miR-197 expression level was significantly correlated with survival time $(\mathrm{P}=0.030)$, and that the patients with higher expression of miR-197 survived for a longer period of time. The Cox single-factor related risk analysis results showed that patient prognosis $(\mathrm{P}=0.000)$, TNM stage $(\mathrm{P}=0.041)$, post-operation $\mathrm{T}(\mathrm{P}=0.010)$, and tumor size $(\mathrm{P}=0.006)$, were significantly correlated with patient survival time. Cox multi-factor model analysis showed that patient prognosis $(\mathrm{P}=0.001)$, tumor length $(\mathrm{P}=0.010)$, and miR-197 expression $(\mathrm{P}=0.042)$ were significantly correlated with survival time, with corresponding risks of 9.183, 2.318, and 1.925, respectively (Figure 2).

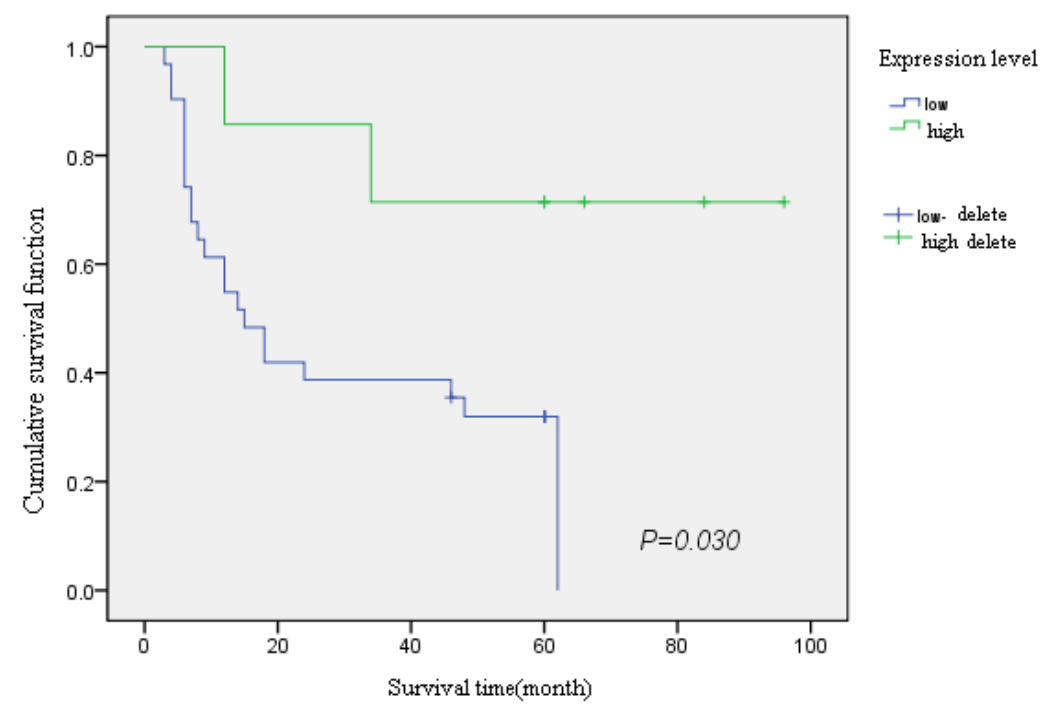

Figure 2. Relationship between miR-197 expression and survival time.

\section{DISCUSSION}

EC patients at the same pathological stage that received the same surgical therapy by the same surgeon showed distinct prognoses. Whether differences in miRNA expression among patients could contribute to different postoperative prognoses remains to be explored.

The potential of miRNAs as novel biomarkers is increasing as more studies report the relationship between miRNAs and cancers (Zoon et al., 2009; Yu et al., 2010). However, there are no reports on miR-197 in EC. In the present study, we found that miR-197 expression was the same among patients at the same pathological stage. However, among patients with varying prognoses, miR-197 expression was higher in the sample with good prognoses and lower in the sample with bad prognoses. Moreover, statistical analysis showed that mir-197 expression was significantly correlated with survival time.

Consistent with our results, previous studies found that miR-197 expression is decreased in cancers, including astrocytoma (Yang et al., 2013a) and oral squamous cell car- 
cinoma (Scapoli et al., 2010). This is explained by its function as an oncogene in which it inhibits tumor suppressor genes. FUS1 is a tumor suppressor gene located on human chromosome $3 \mathrm{p} 21$, and expression of the Fus 1 protein is highly regulated at various levels, leading to lost or greatly diminished tumor suppressor function in many lung cancers. Du et al. (2009) found that elevated miR-93 and miR-197 expression is correlated with reduced Fus 1 expression in non-small cell lung cancer (NSCLC) tumor specimens, suggesting that the 3 miRNAs are negative regulators of Fus 1 expression in lung cancers. Hamada et al. (2013) assessed the difference in miRNA expression profiles of invasive ductal adenocarcinoma (IDA) and intraductal papillary mucinous adenoma or carcinoma, and identified miR-197 as an upregulated miRNA specifically in IDA. Expression of miR-197 in pancreatic cancer cells resulted in the induction of epithelial-mesenchymal transition along with downregulation of p120 catenin, which is a putative target of miR-197. In situ hybridization of miR-197 and immunohistochemistry of p120 catenin showed mutually exclusive patterns, suggesting a pivotal role of miR-197 in the regulation of p120 catenin (Hamada et al., 2013). Other studies reported that miR-197 expression was significantly elevated compared with controls. Zheng et al. (2011) determined miRNAs levels using real-time RT-PCR in 74 lung cancer patients and 68 agematched cancer-free controls, and found that the levels of miR-155, miR-197, and miR-182 in the plasma of lung cancer, including stage I patients, were significantly elevated compared with controls, which may have been related to the serum sample. However, there have been no reports regarding miR-197 and EC.

We confirmed the clinical importance of miR-197 expression by showing its association with unfavorable clinicopathologic features. We found that lower expression of miR197 leads to increased inhibition of tumor suppressor genes, resulting in increased tumor cell proliferation and inhibition of apoptosis; this explains the results of our study, including the correlation with poor prognoses.

This study has several limitations. Although patient selection was consecutive from 2006 to 2009, lack of materials resulted in a small population for analysis and selection bias may have occurred. Because of the small population and short follow-up period, the number of deaths was low, which may have influenced the survival analysis. In addition, there is currently no international standardized definition for methods of analysis and expression levels for miRNAs, and some studies use mean relative quantification values to define high and low expression, while other studies have different definitions for grouping expression levels. This discordant analysis can lead to disagreement among studies.

In conclusion, we found that miR-197 expression is downregulated in EC with poor prognosis, and that lower expression was associated with poor clinicopathological factors and poor survival. These findings suggest that miR-197 has profound potential as a biomarker to predict the outcome and treatment response, as well as a method for treating EC. To clarify the role of miR-197 for its use as a biomarker and in targeted therapy, large, worldwide population-based studies with a standardized definition of miR-197 expression level are necessary.

\section{REFERENCES}

Ambros V (2004). The functions of animal microRNAs. Nature 431: 350-355.

Bagga S, Bracht J, Hunter S, Massirer K, et al. (2005). Regulation by let-7 and lin-4 miRNAs results in target mRNA degradation. Cell 122: 553-563.

Du L, Schageman JJ, Subauste MC, Saber B, et al. (2009). miR-93, miR-98, and miR-197 regulate expression of tumor 
suppressor gene FUS1. Mol. Cancer Res. 7: 1234-1243.

Filipowicz W, Bhattacharyya SN and Sonenberg N (2008). Mechanisms of post-transcriptional regulation by microRNAs: are the answers in sight? Nat. Rev. Genet. 9: 102-114.

Guo Y, Chen Z, Zhang L, Zhou F, et al. (2008). Distinctive microRNA profiles relating to patient survival in esophageal squamous cell carcinoma. Cancer Res. 68: 26-33.

Hamada S, Satoh K, Miura S, Hirota M, et al. (2013). miR-197 induces epithelial-mesenchymal transition in pancreatic cancer cells by targeting p120 catenin. J. Cell Physiol. 228: 1255-1263.

He B, Pan Y, Cho WC, Xu Y, et al. (2012). The association between four genetic variants in microRNAs (rs11614913, rs2910164, rs3746444, rs2292832) and cancer risk: evidence from published studies. PLoS One 7: e49032.

Hobert O (2008). Gene regulation by transcription factors and microRNAs. Science 319: 1785-1786.

Kim VN (2005). MicroRNA biogenesis: coordinated cropping and dicing. Nat. Rev. Mol. Cell Biol. 6: 376-385.

Lee RC, Feinbaum RL and Ambros V (1993). The C. elegans heterochronic gene lin-4 encodes small RNAs with antisense complementarity to lin-14. Cell 75: 843-854.

Liang M (2009). MicroRNA: a new entrance to the broad paradigm of systems molecular medicine. Physiol. Genomics 38: 113-115.

Liang M, Liu Y, Mladinov D, Cowley AW, Jr., et al. (2009). MicroRNA: a new frontier in kidney and blood pressure research. Am. J. Physiol. Renal Physiol. 297: F553-F558.

Lim LP, Lau NC, Garrett-Engele P, Grimson A, et al. (2005). Microarray analysis shows that some microRNAs downregulate large numbers of target mRNAs. Nature 433: 769-773.

Lin RJ, Xiao DW, Liao LD, Chen T, et al. (2012). MiR-142-3p as a potential prognostic biomarker for esophageal squamous cell carcinoma. J. Surg. Oncol. 105: 175-182.

Mathé EA, Nguyen GH, Bowman ED, Zhao Y, et al. (2009). MicroRNA expression in squamous cell carcinoma and adenocarcinoma of the esophagus: associations with survival. Clin. Cancer Res. 15: 6192-6200.

Meister G (2007). miRNAs get an early start on translational silencing. Cell 131: 25-28.

Scapoli L, Palmieri A, Lo ML, Pezzetti F, et al. (2010). MicroRNA expression profiling of oral carcinoma identifies new markers of tumor progression. Int. J. Immunopathol. Pharmacol. 23: 1229-1234.

Sobin LH, Gospodarowicz MK and Wittekind C (2002). TNM Classification of Malignant Tumours (UICC International Union Against Cancer). Wiley-Blackwell Publish House, New York.

Xu X, Chen Z, Zhao X, Wang J, et al. (2012). MicroRNA-25 promotes cell migration and invasion in esophageal squamous cell carcinoma. Biochem. Biophys. Res. Commun. 421: 640-645.

Yang C, Wang C, Chen X, Chen S, et al. (2013a). Identification of seven serum microRNAs from a genome-wide serum microRNA expression profile as potential noninvasive biomarkers for malignant astrocytomas. Int. J. Cancer 132: 116-127.

Yang M, Liu R, Sheng J, Liao J, et al. (2013b). Differential expression profiles of microRNAs as potential biomarkers for the early diagnosis of esophageal squamous cell carcinoma. Oncol. Rep. 29: 169-176.

Yu Z, Baserga R, Chen L, Wang C, et al. (2010). microRNA, cell cycle, and human breast cancer. Am. J. Pathol. 176: 1058-1064.

Zheng D, Haddadin S, Wang Y, Gu LQ, et al. (2011). Plasma microRNAs as novel biomarkers for early detection of lung cancer. Int. J. Clin. Exp. Pathol. 4: 575-586.

Zhou ZQ, Cao WH, Xie JJ, Lin J, et al. (2009). Expression and prognostic significance of THBS1, Cyr61 and CTGF in esophageal squamous cell carcinoma. BMC Cancer 9: 291.

Zoon CK, Starker EQ, Wilson AM, Emmert-Buck MR, et al. (2009). Current molecular diagnostics of breast cancer and the potential incorporation of microRNA. Expert. Rev. Mol. Diagn. 9: 455-467. 